\title{
Marginal populations under pressure: spatial and temporal heterogeneity of Ascophyllum nodosum and associated assemblages affected by human trampling in Portugal
}

\author{
Iacopo Bertocci ${ }^{1, *}$, Rita Araújo ${ }^{1}$, Stefano Vaselli ${ }^{1}$, Isabel Sousa-Pinto ${ }^{1,2}$ \\ ${ }^{1}$ CIIMAR, Centro Interdisciplinar de Investigação Marinha e Ambiental, Rua dos Bragas, 289, 4050-123 Porto, Portugal \\ ${ }^{2}$ Department of Botany, Faculty of Sciences, University of Porto, Rua do Campo Alegre s/n, 1191, 4169-007 Porto, Portugal
}

\begin{abstract}
Anthropogenic disturbances are major threats to coastal biodiversity and may exert drastic effects on natural populations occurring at the borders of the geographical distribution of species, which are thus already experiencing sub-optimal environmental conditions. In this paper, we examined the effects of experimental intensities of human trampling on temporal and spatial variance of the brown seaweed Ascophyllum nodosum and associated organisms in north Portugal. This is the southernmost population of A. nodosum in Europe and shows morphological and demographic traits that are different from those of populations from more central locations within its range of distribution. Results indicated that high trampling intensity reduced the smallscale spatial heterogeneity in the abundance of A. nodosum, whose cover was stabilized around relatively low values, and of the associated red algae Polysiphonia lanosa and Rhodothamniella floridula; however, it increased the spatial variance of limpets and green foliose macroalgae of the genus Ulva. Idiosyncratic responses in temporal patterns were also documented, with high trampling intensity causing relatively larger fluctuations in the structure of whole A. nodosum associated assemblages and in the abundance of Ulva spp., and smaller fluctuations in the cover of Fucus vesiculosus. The present findings are key to predicting the relatively unstudied responses of coastal populations of $A$. nodosum to increasing anthropogenic disturbance and have important implications for the management and conservation of the examined population, which represents a relevant component of the biodiversity of European rocky shores.
\end{abstract}

KEY WORDS: Ascophyllum nodosum - Trampling disturbance $\cdot$ Marginal populations $\cdot$ Spatial heterogeneity $\cdot$ Temporal heterogeneity $\cdot$ Rocky intertidal

\section{INTRODUCTION}

Examining the factors responsible for patterns of distribution, abundance and diversity of organisms is a key issue in ecology (Menge \& Sutherland 1987, Danielson 1991). This is of great concern under current increasing rates of direct and indirect anthropogenic perturbations over terrestrial and marine systems, which are known to drastically affect the geographic limits and range shifts of species (Parmesan et al. 2005, Gaston 2009).

Natural and anthropogenic disturbances are among the main drivers of the structure of natural assemblages through direct and indirect mechanisms (Sousa 1984, Petraitis et al. 1989). Disturbance can directly eliminate some species, but can also release 
new resources that can increase the local abundance of other species (Connell 1978, Huston 1994). Consequently, the ability of assemblages to resist or recover from disturbance depends largely on the particular life traits of each species (Keough \& Quinn 1998, Schiel \& Taylor 1999, Bertocci et al. 2005).

A large number of conceptual and empirical studies have examined the effects of disturbance on the diversity of populations and assemblages, usually focusing on average response variables (McCabe \& Gotelli 2000, Mackey \& Currie 2001, Molinos \& Donohue 2010). In contrast, relatively fewer studies have experimentally investigated the relationships between traits of disturbance and spatial and temporal patterns of distribution, abundance and diversity of organisms (Collins 2000, Benedetti-Cecchi et al. 2005, Bertocci et al. 2005). Nevertheless, there is mounting evidence of their ecological importance. Temporal fluctuations of species, for instance, may cause drastic changes in functional traits of natural systems, including productivity and stability (Johnson et al. 1996), and may be related directly to the risk of extinction (Inchausti \& Halley 2003) and inversely to overall biodiversity (Lande 1993, Vucetich et al. 2000). Spatial variability of key organisms (e.g. grazers and predators) may be responsible for patchy distributions of consumed resources (Fairweather 1988, Navarrete 1996, Benedetti-Cecchi et al. 2005). Spatial heterogeneity of abiotic factors (e.g. the availability of nutrients) may drastically affect the outcome of biological interactions, and spatially heterogeneous habitats may support assemblages significantly different to those found in more homogenous habitats (Day et al. 2003).

Recreational uses and commercial harvesting are major anthropogenic sources of disturbance in intertidal habitats (Keough \& Quinn 1998, Brown \& Taylor 1999, Schiel \& Taylor 1999). Intense trampling associated with such activities can drastically affect patterns of distribution, abundance and diversity of macroalgal assemblages on rocky shores (Keough \& Quinn 1998, Araújo et al. 2009).

There is increasing evidence that canopy-forming macroalgae are particularly sensitive to anthropogenic disturbance (Benedetti-Cecchi et al. 2001, Milazzo et al. 2004, Airoldi \& Beck 2007, Connell et al. 2008, Mangialajo et al. 2008). As foundation species, they provide a specific habitat for diversified associated organisms and contribute to the high biodiversity of rocky shores (Pavia et al. 1999, Lilley \& Schiel 2006, Schiel \& Foster 2006). Their reduced abundance may cause drastic changes in associated assemblages through modifications of light condi- tions, temperature, hydrodynamics, sedimentation and habitat preemption (Reed \& Foster 1984, McCook \& Chapman 1991) that may exert positive (e.g. by ameliorating physical conditions), or negative (e.g. by monopolizing the substratum) effects (Bertness et al. 1999, Bulleri et al. 2002). Under current and predicted scenarios of increasing anthropogenic threats to the persistence of canopy-forming macroalgae (e.g. Lilley \& Schiel 2006), investigating their responses and those of associated assemblages is of overwhelming conservation importance.

On temperate rocky shores, common habitat formers are large fucoid algae, such as Ascophyllum nodosum (L.) Le Jol., which are typically dominant in the intertidal habitat on sheltered rocky shores of the North Atlantic (Pavia et al. 1999). The European distribution of $A$. nodosum ranges between the Arctic circle and North Portugal, where a single population occurs in a rocky intertidal area $\sim 3 \mathrm{~km}$ long, and $10 \mathrm{~s}$ of $\mathrm{km}$ from the nearest northern populations (Lima et al. 2007). Previous studies have indicated a variety of abiotic and biological factors and processes as responsible for variations in the patterns of $A$. nodosum and associated assemblages at several locations within its geographical range (Jenkins et al. 1999, Cervin et al. 2005, Svensson et al. 2009). In contrast, experimental studies on the effects of disturbance on marginal populations are rare (but see Araújo et al. 2009).

Marginal populations (i.e. those occurring at peripheral locations corresponding to the limits of their geographical distribution) are assumed to live in a sub-optimal environment (Bridle \& Vines 2007) and thus represent target systems for investigating the consequences of natural and anthropogenic stressors. Such populations often occur in small sizes which, combined with the harshness of local conditions, may reduce their resistance and resilience to further disturbances (Guo et al. 2005). Edge populations may also show distinctive ecological adaptations that confer upon them an important conservation value under scenarios of environmental modifications, such as those predicted by climate models (Hampe \& Petit 2005).

The population of Ascophyllum nodosum on the north Portuguese coast is an excellent system for addressing these issues for several reasons. Firstly, the general life traits of this species, including low recruitment and dispersal ability (Åberg \& Pavia 1997, Dudgeon \& Petraitis 2005) and slow growth (Åberg \& Pavia 1997, Cervin et al. 2005), make it highly sensitive to disturbance (Olsen et al. 2010). Secondly, as a marginal population, it might be ex- 
posed to an increased extinction risk compared to centrally located populations due to its relatively small size and isolation (Guo et al. 2005). Thirdly, this population has a relevant conservation value as it shows distinguishing morphological and demographic traits, including higher reproductive output, higher density and smaller size of individuals compared to central populations located in northwest France (Araújo et al. 2011).

A previous experimental study on the Portuguese population of Ascophyllum nodosum showed that high intensities of human trampling can negatively affect the mean abundance of this species, the cooccurring fucoid Fucus vesiculosus and understorey species, while enhancing the cover of ephemeral green algae (Araújo et al. 2009). However, how changes in the intensity of disturbance affect spatial and temporal patterns of the same population and associated organisms has never been experimentally investigated. The present study was aimed at filling this gap, by experimentally testing hypotheses on the effects of a range of trampling intensities on measures of temporal and spatial variance of $A$. nodosum and associated algal and invertebrate taxa in North Portugal. Theoretical (Benedetti-Cecchi 2003), modelling (Benedetti-Cecchi 2000) and empirical (Berlow 1999, Benedetti-Cecchi et al. 2005, Bertocci et al. 2005) studies suggested that large spatial and temporal variability of response variables may be associated with mild disturbances (low intensity), while reduced fluctuations in space and/or time may be caused by severe (high intensity) disturbance. Such predictions are mostly the consequence of the scaling relationship between the mean and the variance (Taylor 1961), which should apply in all cases where a response variable is drastically reduced, such as the cover of benthic organisms exposed to extreme events of disturbance. Therefore, we specifically tested the hypotheses that (1) for species, such as A. nodosum and other associated fucoid algae, that are directly affected negatively by trampling, increasing intensity of experimental events of human trampling would be inversely related to measures of the temporal and spatial heterogeneity of abundance, while (2) the opposite would occur for species, such as ephemeral green algae, that could indirectly benefit from disturbance. Effects of experimental trampling on (1) the temporal and spatial variation of the total number of taxa (a surrogate for species richness) and (2) the structure of whole assemblages associated with $A$. nodosum resulting from such changes in individual abundances were also examined.

\section{MATERIALS AND METHODS}

\section{Study site and experimental procedures}

This study was carried out between December 2005 and April 2006 on the rocky shore of Praia Norte in Viana do Castelo, NW Portugal $\left(41^{\circ} 41^{\prime} \mathrm{N}\right.$, $8^{\circ} 51^{\prime} \mathrm{W}$ ), on intertidal assemblages dominated by Ascophyllum nodosum, representing the southernmost population in Europe. Abiotic and biological characteristics of the study site have been previously described in detail (Araújo et al. 2009). The shore is subject to intense and frequent human recreational and harvesting activities, particularly during summer (Araújo et al. 2009). Therefore, to experimentally test the effects of trampling on $A$. nodosum assemblages, a manipulative experiment was performed during winter and early spring months and at the less frequented parts of the shore, as indicated in Araújo et al. (2009).

Unmanipulated control and 3 levels of experimental intensity (low, medium and high: LI, MI and HI, corresponding to 5, 15 and 30 tramples, respectively) of trampling were chosen, based on preliminary estimations of the number of people visiting the shore during $1 \mathrm{yr}$ and applied in the field following procedures described in detail in Araújo et al. (2009). Briefly, it was assumed that the trampling disturbance experienced by each experimental patch $(2.5 \times 2.5 \mathrm{~m})$ was directly related to the number of people visiting the shore at low tide, as estimated according to a preliminary survey carried out for a period of $1 \mathrm{yr}$ on 4 stretches of shore ( $100 \mathrm{~m}$ long) interspersed throughout the study site. The LI treatment was then selected as corresponding to the observed annual intensity of trampling averaged across all stretches, MI as corresponding to the average of the 5 highest values of observed frequencies of human presence, and $\mathrm{HI}$ as simulating a further increase $(2 \times \mathrm{MI})$ in the human frequency of human visits to the shore. Experimental disturbance was performed by dividing each experimental patch into 4 rows that were trampled by 2 persons weighing $\sim 70 \mathrm{~kg}$ each and wearing gumboots, with the number of tramples for LI, MI and HI corresponding to the number of times each row was trampled. Two trampling events were carried out each month during each week of spring tides.

The abundance of Ascophyllum nodosum and associated organisms was visually estimated in terms of percentage cover (sessile organisms) or number of individuals (mobile animals) in each of three $50 \times$ $50 \mathrm{~cm}$ quadrats randomly established in each of three $2.5 \times 2.5 \mathrm{~m}$ patches assigned to control and trampling 
intensity treatments (see Araújo et al. 2009 for details) at 5 dates over the experimental period.

\section{Data analysis}

To examine the effects of experimental trampling on the temporal and spatial heterogeneity of the structure of whole assemblages and the abundance of individual taxa, multivariate and univariate measures of variance over the 5 dates of sampling and over replicate quadrats (data from the 3 replicate quadrats per patch per sampling time were first averaged) were calculated separately for each patch assigned to each treatment. Measures of temporal variance of the abundance of the most numerous taxa and of the total number of taxa were calculated by estimating variance components using a 1-way ANOVA with time as a factor, performed separately for each response variable in each patch. This procedure produced replicate estimates of temporal variance independent of sampling error (Searle et al. 1992). Negative values were interpreted as underestimates of null variances and were set to zero. Analogous estimates were calculated for multivariate responses to experimental treatments. Multivariate pseudo-variance components were estimated from Bray-Curtis dissimilarities (Bray \& Curtis 1957), which were calculated on untransformed data separately for each patch, using permutational multivariate analysis of variance (PERMANOVA, Anderson 2001) with time as a factor. Variance and pseudovariance components were then analyzed using a 1-way ANOVA with Intensity as a fixed factor that included 4 levels (unmanipulated control, low, medium and high intensity of trampling) and 3 replicates (the 3 patches assigned to each treatment).

Analogous procedures were used to estimate variance and pseudo-variance components of univariate and multivariate response variables, respectively, among the 3 replicate quadrats sampled per patch per sampling time. These produced independent estimates of spatial variance that were analyzed with a 2-way ANOVA, including the crossed factors Time (random, 5 levels) and Intensity (fixed, 4 levels as in the previous analysis), with 3 replicates.

Before each ANOVA, Cochran's $C$ tests were done to check for homogeneity of variances. When necessary, data were log-transformed. Student-NewmanKeuls (SNK) tests were used for post-hoc comparisons of significant means.

Changes in the structure of whole assemblages in each treatment were graphically compared by calcu- lating centroids of 'average' assemblages per patch per sampling date. First, principal coordinates (Gower 1966) were calculated from the Bray-Curtis dissimilarity matrix of the whole data set. Then, centroids were obtained separately for each treatment and sampling time by averaging the principal coordinates over the 3 replicates per patch (McArdle \& Anderson 2001). Finally, multivariate patterns were displayed using a non-metric multidimensional scaling (nMDS) plot based on Euclidean distances.

\section{RESULTS}

\section{Temporal heterogeneity}

Assemblages exposed to the highest intensity of experimental trampling showed temporal fluctuations that are significantly larger than those occurring in unmanipulated patches and under the 2 lower intensities (Fig. 1; ANOVA: $\mathrm{MS}_{\text {Intensity }}=$ 89 169.9, $F_{3,8}=7.61, \mathrm{p}<0.01$; SNK: $\mathrm{HI}>\mathrm{MI}=$ Control $=\mathrm{LI}, \mathrm{SE}=62.496)$. The only univariate response variables that were significantly affected by trampling were the brown fucoid alga Fucus vesiculosus $\left(\mathrm{MS}_{\text {Intensity }}=94667.7, F_{3,8}=5.18, \mathrm{p}<0.05\right)$ and green foliose algae of the genus Ulva $\left(\mathrm{MS}_{\text {Intensity }}=21.1\right.$, $\left.F_{3,8}=23.28, \mathrm{p}<0.001\right)$, but with clearly different pat-

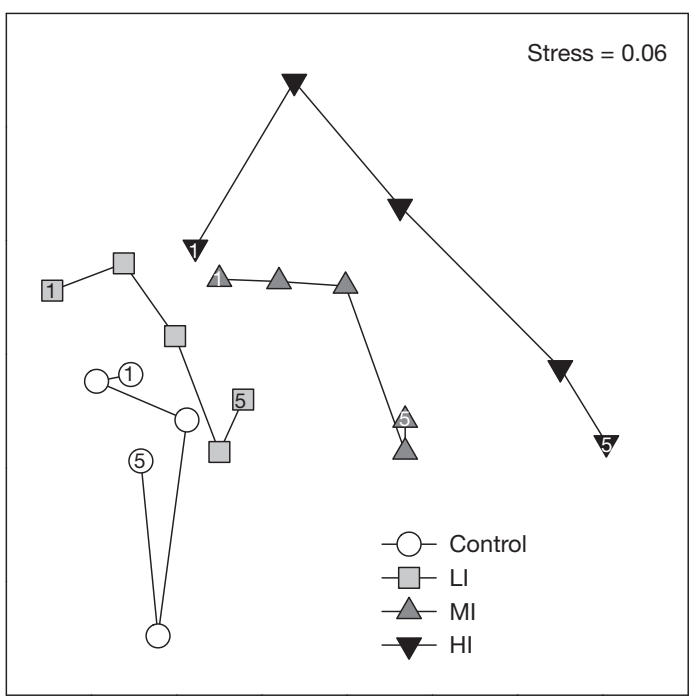

Fig. 1. Ascophyllum nodosum. Non-metric multidimensional scaling (nMDS) of the temporal trajectories of assemblages in the unmanipulated treatment (control) and under different trampling levels (LI, MI and HI: low, medium and high intensity, respectively). Symbols: centroids of assemblages ('averaged' across quadrats and patches) subject to each treatment at each sampling time (1 to 5), and calculated from principal coordinates 
terns. Each trampled treatment caused reduced fluctuations in the abundance of $F$. vesiculosus compared to the control (Fig. 2B; SNK: Control $>\mathrm{MI}=\mathrm{LI}=\mathrm{HI}$, $\mathrm{SE}=78.054$ ), while the abundance of Ulva spp. was more variable during the study under the 2 highest intensities of experimental disturbance than under the low intensity and in unmanipulated patches (Fig. 2G; SNK: HI = MI > LI = Control). All other individual taxa examined, including Ascophyllum nodosum, and the total number of taxa did not respond significantly to trampling, and showed temporal variations over the course of the study that are comparable among treatments despite large differences in abundances (Fig. 2A,C-F,H-J). canopy-forming species. Effects of treatments did not vary with time of sampling for all multivariate and univariate response variables (Tables $1 \& 2$ ). The spatial heterogeneity of $A$. nodosum was larger in the control than under medium and high trampling intensity, with low intensity being inconsistently ranked between these 2 treatments (Table 2, Fig. 4A). Similar patterns were displayed by the turf-forming red algae Polysiphonia lanosa, with consistently lower values for all manipulated treatments compared to the control (Table 2, Fig. 4F), and by Rhodothamniella floridula, whose spatial heterogeneity was larger in unmanipulated patches and under low disturbance than under both more severely trampled treatments

\section{Spatial heterogeneity}

Trampling did not affect the spatial variability of the structure of assemblages at the scale of quadrats (Table 1, Fig. 3) and the total number of taxa (Table 2, Fig. 4J), while it exerted significant effects on Ascophyllum nodosum and a number of individual taxa associated with this
Table 1. ANOVA examining the effects of time and intensity of trampling on spatial variance of the structure of assemblages. ns: not significant ( $p>0.05$ )

\begin{tabular}{|lcccc|}
\hline Source of variation & df & MS & $F$ & Denominator for $F$ \\
\hline Time, T & 4 & 0.26 & 0.65 & Residual \\
Intensity, I & 3 & 0.87 & 2.51 & T $\times$ I \\
T $\times$ I & 12 & 0.35 & 0.87 & Residual \\
Residual & 40 & 0.40 & & \\
Cochran's $C$ test & $C=0.136, \mathrm{~ns}$ & & & \\
Transformation & $\mathrm{Ln}(\mathrm{x}+1)$ & & & \\
\hline
\end{tabular}

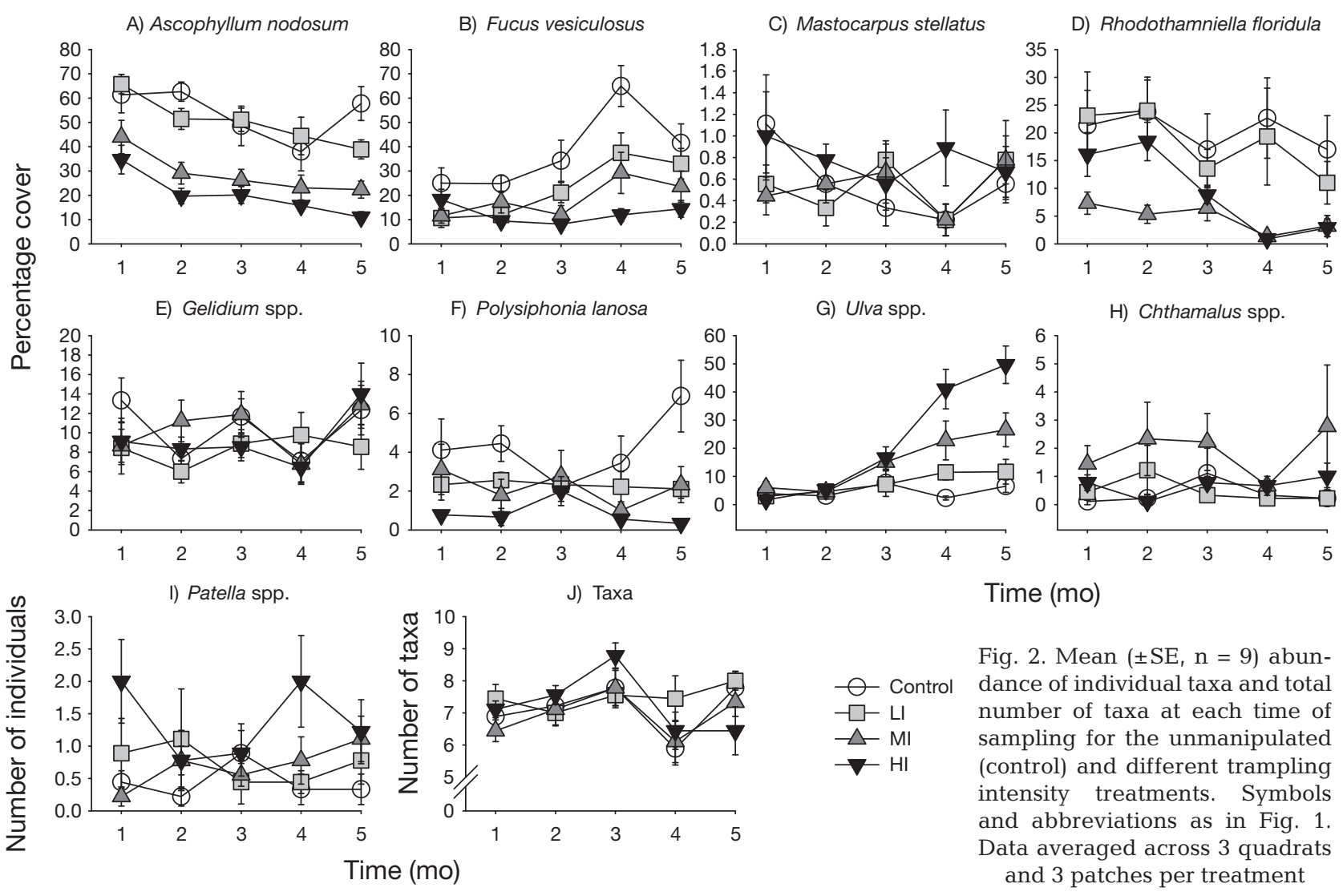




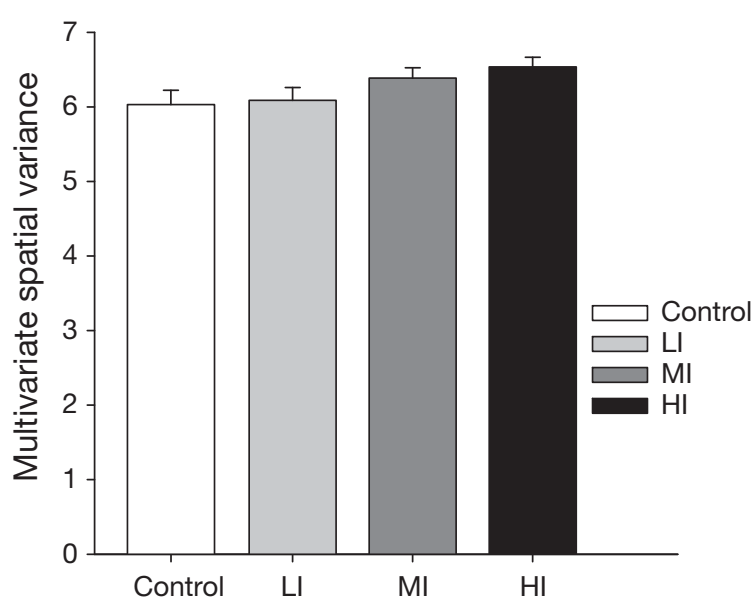

Fig. 3. Ascophyllum nodosum. Spatial variance (mean $\pm \mathrm{SE}$, $\mathrm{n}=15$, on a logarithmic scale) of the structure of assemblages in the unmanipulated (control) and different trampling intensity treatments. Abbreviations are as in Fig. 1. Data averaged across 3 patches and 5 sampling dates

(Table 2, Fig. 4D). Conversely, high disturbance intensity was associated with larger spatial heterogeneity of grazing limpets of the genus Patella, compared to the other manipulated treatments and the control (Table 2, Fig. 4I). Similarly, the small-scale distribution of Ulva spp. was more heterogeneous under very intense trampling than in unmanipulated patches, with the other treatments being inconsistently ranked (Table 2, Fig. 4G). Ulva spp. was the only taxon showing significant differences among sampling times independently of treatments (Table 2). No significant effects were documented for all other macroalgae (Table 2, Fig. 4B,C,E) and animals (Table 2, Fig. 4H) examined.

\section{DISCUSSION}

According to the well documented positive relationship between the mean and the variance in the abundance of natural populations (Taylor 1961, McArdle \& Gaston 1992, Gaston \& McArdle 1993), we hypothesized that experimental treatments that are able to decrease individual abundances of taxa to very low values would also cause drastic reductions in their temporal and spatial measures of variability. Conversely, larger heterogeneity in space and time would be possible for taxa responding positively to the disturbance applied, in agreement with the expected differential effects of disturbance depending on taxon-specific life traits of organisms (e.g. Bertocci et al. 2005). Findings basically consistent

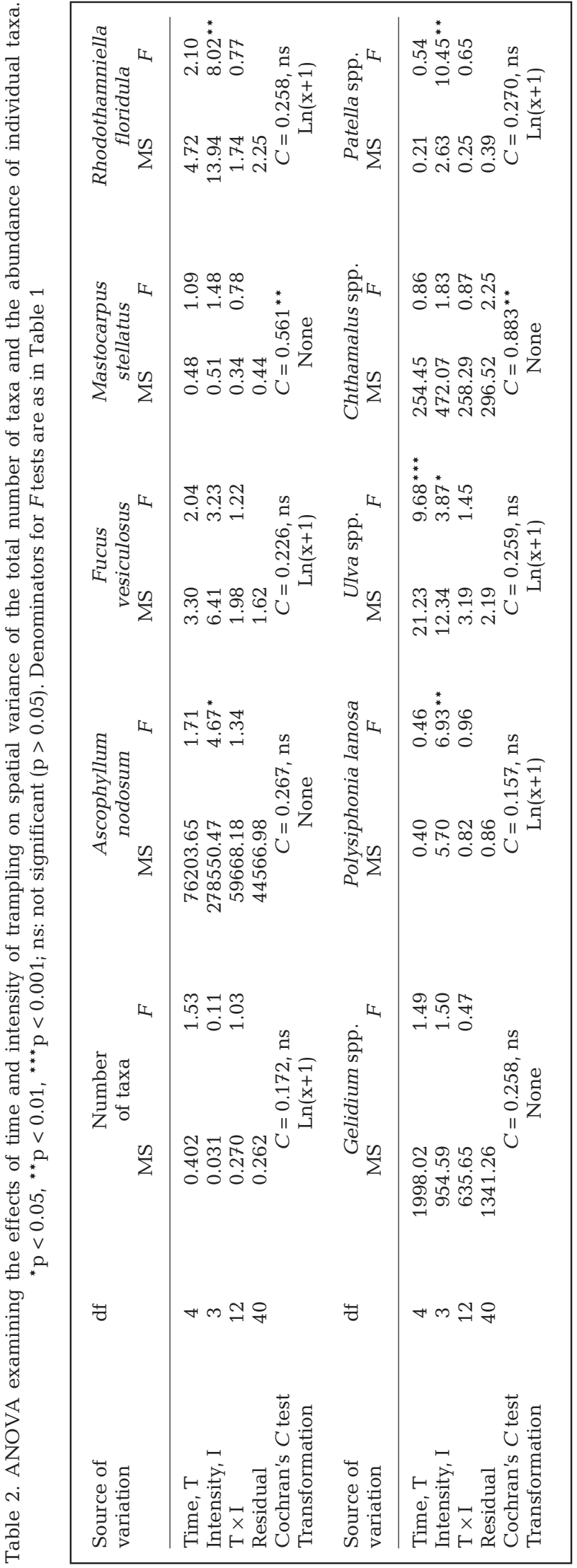




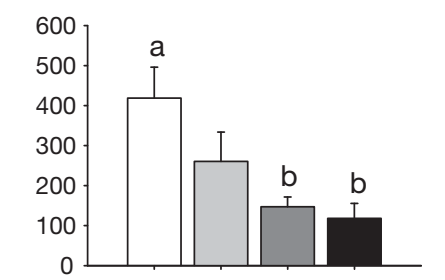

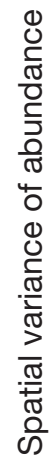

E) Gelidium spp.

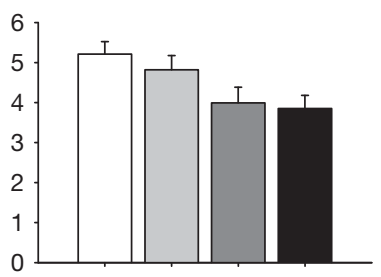

F) Polysiphonia lanosa

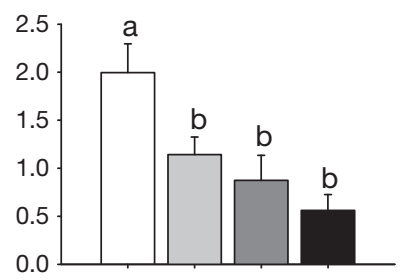

J) Taxa
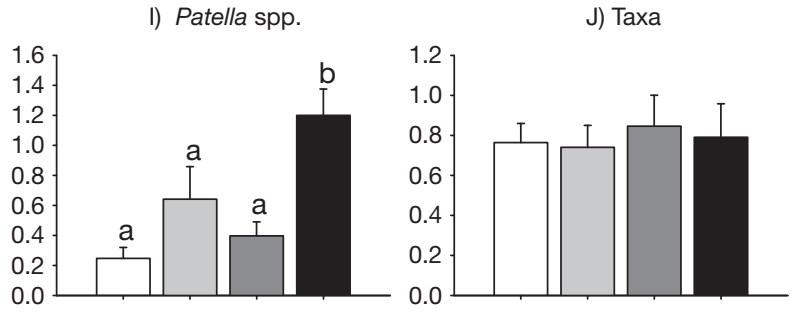

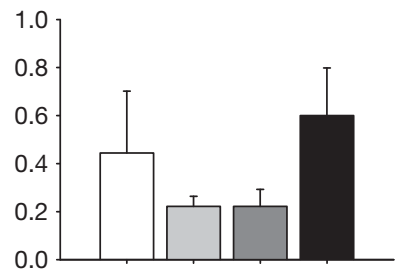

G) Ulva spp.
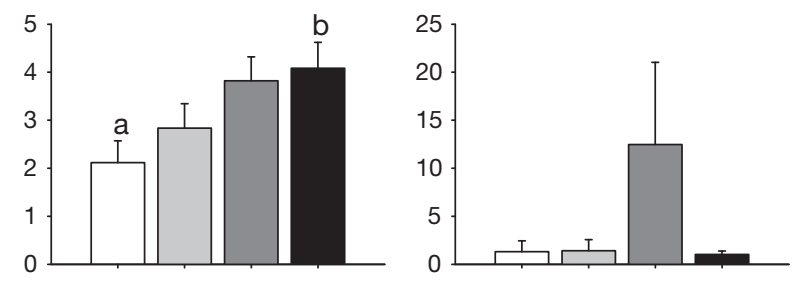

Fig. 4. Spatial variance (mean $\pm \mathrm{SE}, \mathrm{n}=15$ ) of the abundance of individual taxa and the total number of taxa in the unmanipulated (control) and different trampling intensity treatments. Note that $\mathrm{A}, \mathrm{C}, \mathrm{E}$ and $\mathrm{H}$ are untransformed, while $\mathrm{B}$, D, F, G, I and J are on a logarithmic scale. Symbols and abbreviations are as in Fig. 1. Data averaged across 3 patches and 5 sampling dates. Different lowercase letters above bars indicate treatments that differ significantly at $\mathrm{p}<0.05$ (Student-Newman-Keuls tests) with such predictions were obtained for temporal patterns in the abundance of Fucus vesiculosus and the ephemeral green algae of the genus Ulva and for spatial patterns in the abundance of Ascophyllum nodosum, the turf-forming algae Polysiphonia lanosa and Rhodothamniella floridula, limpets and Ulva spp. The other response variables examined were generally not affected by trampling.

The small-scale spatial heterogeneity of Ascophyllum nodosum was negatively affected by the medium and high intensity of trampling, which also drastically reduced the abundance of this species throughout the experiment compared to the less or undisturbed treatments. Such a response was likely due to the mechanical impact of trampling on the canopy, which removed plants and generated haloes of bare space that increase in size with increasing intensity of disturbance. This led to an increasing spatial homogenization of the distribution of this alga, which levelled off around low abundance values. Although specifically examining long-term responses to trampling is beyond the goals of the present study, we could also hypothesize that the new spatial patterns might further compromise the recovery of the canopy due to species and population specific traits of
A. nodosum. It has been documented that due to its low dispersal abilities, the growth and expansion of this species mostly depend on the development of recruits established under the canopy created by adults of the same species (Åberg \& Pavia 1997, Cervin et al. 2005); this canopy provides protection against excessive temperatures and hydrodynamics (Vadas et al. 1990) and prevents the establishment of potential competitors for space (Dudgeon \& Petraitis 2001). By creating patches of bare space, disturbance may, therefore, drastically prevent recolonization by A. nodosum as the distance from parental algae increases - an effect that would be proportional to the intensity of disturbance. A similar mechanism was considered responsible for the occurrence of alternative communities on the rocky shores of Maine, where intertidal areas subject to low disturbance are dominated by Ascophyllum, which are able to recolonize the small patches produced by disturbance despite its very limited dispersal; areas with a history of larger disturbances are colonized by mussels and barnacle species with relatively wider dispersal abilities (Petraitis \& Dudgeon 1999). Moreover, the potential negative effects of the spatial patterns of distribution of $A$. nodosum determined by 
disturbance might be exacerbated by the isolation of the studied marginal population from nearby sources of propagules (Guo et al. 2005). Conversely, where A. nodosum was more abundant, such as in control patches, a larger spatial heterogeneity could have been generated and maintained by abiotic and biological processes, including wave action (Vadas et al. 1990) and grazing (Pavia \& Toth 2000, Davies et al. 2007). In principle, we could have obtained analogous results for the temporal variability of $A$. nodosum, but this was not the case, as this response variable was comparable among all trampling intensities and not different from the unmanipulated condition. In fact, the abundance of this species during the experiment was generally stable under all experimental conditions, either where percentage cover values were relatively low due to severe (MI and $\mathrm{HI}$ ) disturbance, or large due to mild (LI) or no (control) disturbance.

Several studies have documented large changes in associated assemblages after natural (e.g. Davies et al. 2007), anthropogenic (e.g. Keser et al. 1981, Ang et al. 1996) and experimental (e.g. Jenkins et al. 1999, Viejo et al. 1999, Dudgeon \& Petraitis 2001, Bertness et al. 2002) disturbances affecting Ascophyllum nodosum. Species occurring in close association with $A$. nodosum are particularly expected to show patterns of variability analogous to those of the canopy-forming species (Cervin et al. 2005). In the present study, this clearly occurred for the turfforming red algae Polysiphonia lanosa and Rhodothamniella floridula, the former being an obligate epiphyte of $A$. nodosum (and, more rarely, of other fucoids), and the latter being a typical understory species of large brown seaweeds (Hayward et al. 1996). Nevertheless, corresponding variations were not documented for spatial and temporal patterns in the structure of the whole assemblage, the total number of taxa and the abundance of most of the other A. nodosum associated organisms, as such variables were generally not affected by trampling. For Gelidium spp., results agree with the previously documented resistance of algal turfs to trampling and are likely due to their morphology (Brosnan \& Crumrine 1994, Schiel \& Taylor 1999, Milazzo et al. 2004, Goodsell \& Underwood 2008). More surprising was the lack of significant responses of Chthamalus spp. and the red macroalga Mastocarpus stellatus, as Brosnan \& Crumrine (1994) reported relevant negative effects of trampling on both barnacles and algae of the same genus. It is worth noting, however, that the intensity of experimental trampling applied by those authors was approximately double the most severe disturbance applied here (Brosnan \& Crumrine 1994, Araújo et al. 2009) and, therefore, might have been more effective in damaging these taxa.

High trampling intensity was associated with relatively higher mean abundance and spatial and/or temporal heterogeneity of limpets and Ulva spp. On the contrary, large fluctuations of limpets in space did not occur in unmanipulated patches and in treatments (LI and MI) where they were relatively less abundant, and cover values of algal turfs, such as Rhodothamniella floridula (the most abundant filamentous species here), were larger. It is known that grazing invertebrates tend to be excluded from substrates monopolized by algal turfs (Underwood \& Jernakoff 1981), while they can be favoured by relatively large gaps of free space made available by intense disturbance (e.g. Bertocci et al. 2005), such as in the HI treatment here. Patterns of distribution of limpets could have affected those of the grazed Ulva spp. in the same direction. There is evidence that changes in spatial or temporal variance of the abundance of consumers may drastically affect patterns of resources (Berlow 1999, Benedetti-Cecchi 2000, Benedetti-Cecchi et al. 2005). In addition, the genus Ulva includes opportunistic species that typically attain large cover values on rocky shores only where the abundance of potential competitors for space is reduced (e.g. Denny \& Gaines 2007). These ecological mechanisms could have contributed to maintaining the low cover of these algae in undisturbed or less disturbed treatments, where canopy-forming species such as Ascophyllum nodosum and Fucus vesiculosus were conversely more abundant.

The present findings indicate that intense anthropogenic disturbance may cause homogenization of spatial patterns of distribution of the population of Ascophyllum nodosum in North Portugal, with cover being stabilized around low values and its possible replacement by opportunistic algae. Although this population shows life-history traits that likely resulted in adaptation, on evolutionary scales, to sub-optimal conditions (Araújo et al. 2011), the increasing intensity and rates of human use of the studied shore may represent a relevant extinction threat. Under such circumstances, for instance, a further reduced and isolated population might not be able to respond efficiently to more extreme environmental conditions, such as those due to predicted climate change. This highlights the need for management and protection measures that would allow the conservation of the distinctive traits of this peripheral $A$. nodosum population and the preservation of an important component of the biodiversity of European rocky shores. 
Acknowledgements. We thank E. Maggi and 3 anonymous reviewers for insightful comments on the manuscript. This study was funded by a project (POCI/MAR/56149/2004) and fellowships (to R.A. and S.V.) from the Portuguese Fundação para a Ciência e a Tecnologia (FCT) through the program POCI 2010, with the support of FEDER and FSE. I.B. was supported by FCT within the Programa Ciência 2008Fundo Social Europeu.

\section{LITERATURE CITED}

Åberg P, Pavia H (1997) Temporal and multiple scale spatial variation in juvenile and adult abundance of the brown alga Ascophyllum nodosum. Mar Ecol Prog Ser 158: 111-119

Airoldi L, Beck MW (2007) Loss, status and trends for coastal marine habitats of Europe. Oceanogr Mar Biol Annu Rev 45:345-405

Anderson MJ (2001) A new method for non-parametric multivariate analysis of variance. Austral Ecol 26:32-46

> Ang PO, Sharp GJ, Semple RE (1996) Comparison of the structure of populations of Ascophyllum nodosum (Fucales, Phaeophyta) at sites with different harvesting histories. Hydrobiologia 326-327:179-184

- Araújo R, Vaselli S, Almeida M, Serrão E, Sousa-Pinto I (2009) Effects of disturbance on marginal populations: human trampling on Ascophyllum nodosum assemblages at its southern distribution limit. Mar Ecol Prog Ser 378:81-92

Araújo R, Serrão E, Sousa-Pinto I, Åberg P (2011) Phenotypic differentiation at southern limit borders: the case study of two fucoid macroalgal species with different life-history traits. J Phycol 47:451-462

Benedetti-Cecchi L (2000) Variance in ecological consumerresource interactions. Nature 407:370-374

> Benedetti-Cecchi L (2003) The importance of the variance around the mean effect size of ecological processes. Ecology 84:2335-2346

Benedetti-Cecchi L, Pannacciulli F, Bulleri F, Moschella PS, Airoldi L, Relini G, Cinelli F (2001) Predicting the consequences of anthropogenic disturbance: large-scale effects of loss of canopy algae on rocky shores. Mar Ecol Prog Ser 214:137-150

Benedetti-Cecchi L, Vaselli S, Maggi E, Bertocci I (2005) Interactive effects of spatial variance and mean intensity of grazing on algal cover in rock pools. Ecology 86: 2212-2222

Berlow EL (1999) Strong effects of weak interactions in ecological communities. Nature 398:330-334

> Bertness MD, Leonard GH, Levine JM, Schmidt PR, Ingraham AO (1999) Testing the relative contribution of positive and negative interactions in rocky intertidal communities. Ecology 80:2711-2726

> Bertness MD, Trussell GC, Ewanchuk PJ, Silliman BR (2002) Do alternate stable community states exist in the Gulf of Maine rocky intertidal zone? Ecology 83:3434-3448

> Bertocci I, Maggi E, Vaselli S, Benedetti-Cecchi L (2005) Contrasting effects of mean intensity and temporal variation of disturbance on assemblages of rocky shores. Ecology 86:2061-2067

Bray JR, Curtis JT (1957) An ordination of the upland forest communities of Southern Wisconsin. Ecol Monogr 27: 325-349

Bridle JR, Vines TH (2007) Limits to evolution at range mar- gins: when and why does adaptation fail? Trends Ecol Evol 22:140-147

Brosnan DM, Crumrine LL (1994) Effects of human trampling on marine rocky shore communities. J Exp Mar Biol Ecol 177:79-97

Brown PJ, Taylor RB (1999) Effects of trampling by humans on animals inhabiting coralline algal turf in the rocky intertidal. J Exp Mar Biol Ecol 235:45-53

$>$ Bulleri F, Benedetti-Cecchi L, Acunto S, Cinelli F, Hawkins SJ (2002) The influence of canopy algae on vertical patterns of distribution of low-shore assemblages on rocky coasts in the northwest Mediterranean. J Exp Mar Biol Ecol 267:89-106

Cervin G, Åberg P, Jenkins SR (2005) Small-scale disturbance in a stable canopy dominated community: implications for macroalgal recruitment and growth. Mar Ecol Prog Ser 305:31-40

Collins SL (2000) Disturbance frequency and community stability in native tallgrass prairie. Am Nat 155:311-325

> Connell JH (1978) Diversity in tropical rain forests and coral reefs. Science 199:1302-1310

> Connell SD, Russell BD, Turner DJ, Sheperd SA and others (2008) Recovering a lost baseline: missing kelp forest from a metropolitan coast. Mar Ecol Prog Ser 360:63-72

> Danielson BJ (1991) Communities in a landscape: the influence of habitat heterogeneity on the interactions between species. Am Nat 138:1105-1120

> Davies AJ, Johnson MP, Maggs CA (2007) Limpet grazing and loss of Ascophyllum nodosum canopies on decadal time scales. Mar Ecol Prog Ser 339:131-141

Day KJ, Hutchings MJ, John EA (2003) The effects of spatial pattern of nutrient supply on yield, structure and mortality in plant populations. J Ecol 91:541-553

Denny MW, Gaines SD (2007) Encyclopedia of tidepools and rocky shores. University of California Press, Berkeley, CA

> Dudgeon S, Petraitis PS (2001) Scale-dependent recruitment and divergence of intertidal communities. Ecology 82: 991-1006

Dudgeon S, Petraitis PS (2005) First year demography of the foundation species, Ascophyllum nodosum, and its community implications. Oikos 109:405-415

> Fairweather PG (1988) Predation creates haloes of bare space among prey on rocky seashores in New South Wales. Aust J Ecol 13:401-409

Gaston KJ (2009) Geographic range limits: achieving synthesis. Proc Biol Sci 276:1395-1406

> Gaston KJ, McArdle BH (1993) Measurement of variation in the size of populations in space and time: some points of clarification. Oikos 68:357-360

Goodsell PJ, Underwood AJ (2008) Complexity and idiosyncrasy in the responses of algae to disturbance in monoand multi-species assemblages. Oecologia 157:509-519

Gower JC (1966) Some distance properties and latent root and vector methods used in multivariate analysis. Biometrika 53:325-338

> Guo QF, Taper M, Schoenberger M, Brandle J (2005) Spatial-temporal population dynamics across species range: from centre to margin. Oikos 108:47-57

> Hampe A, Petit RJ (2005) Conserving biodiversity under climate change: the rear edge matters. Ecol Lett 8:461-467

Hayward P, Nelson-Smith T, Shields C (1996) Seashore of Britain and Europe. Harper Collins Publishers, London

Huston M (1994) Biological diversity. Cambridge University Press, Cambridge 
Inchausti P, Halley J (2003) On the relation between temporal variability and persistence time in animal populations. J Anim Ecol 72:899-908

> Jenkins SR, Hawkins SJ, Norton TA (1999) Direct and indirect effects of a macroalgal canopy and limpet grazing in structuring a sheltered inter-tidal community. Mar Ecol Prog Ser 188:81-92

> Johnson KH, Vogt KH, Clark HJ, Schmitz OJ, Vogt DJ (1996) Biodiversity and the productivity and stability of ecosystems. Trends Ecol Evol 11:372-377

Keough MJ, Quinn GP (1998) Effects of periodic disturbances from trampling on rocky intertidal algal beds. Ecol Appl 8:141-161

Keser M, Vadas RL, Larson BR (1981) Regrowth of Ascophyllum nodosum and Fucus vesiculosus under various harvesting regimes in Maine, USA. Bot Mar 24:29-38

- Lande R (1993) Risk of population extinction from demographic and environmental stochasticity and random catastrophes. Am Nat 142:911-927

Lilley SA, Schiel DR (2006) Community effects following the deletion of a habitat forming alga from rocky marine shores. Oecologia 148:672-681

Lima FP, Ribeiro PA, Queiroz N, Hawkins SJ, Santos AM (2007) Do distributional shifts of northern and southern species of algae match the warming pattern? Glob Change Biol 13:1-13

Mackey RL, Currie DJ (2001) The diversity-disturbance relationship: is it generally strong and peaked? Ecology 82:3479-3492

Mangialajo L, Chiantore M, Cattaneo-Vietti R (2008) Loss of fucoid algae along a gradient of urbanisation, and structure of benthic assemblages. Mar Ecol Prog Ser 358:63-74

> McArdle BH, Anderson MJ (2001) Fitting multivariate models to community data: a comment on distance-based redundancy analysis. Ecology 82:290-297

McArdle BH, Gaston KJ (1992) Comparing population variabilities. Oikos 64:610-612

> McCabe DJ, Gotelli NJ (2000) Effects of disturbance frequency, intensity, and area on assemblages of stream invertebrates. Oecologia 124:270-279

> McCook LJ, Chapman ARO (1991) Community succession following massive ice-scour on an exposed rocky shore: effects of Fucus canopy algae and of mussels during late succession. J Exp Mar Biol Ecol 154:137-169

Menge BA, Sutherland JP (1987) Community regulation: variation in disturbance, and predation in relation to environmental stress and recruitment. Am Zool 130: 730-757

Milazzo M, Badalamenti F, Riggio S, Chemello R (2004) Patterns of algal recovery and small-scale effects of canopy removal as a result of human trampling on a Mediterranean rocky shallow community. Biol Conserv 117: 191-202

Molinos JG, Donohue I (2010) Interactions among temporal patterns determine the effects of multiple stressors. Ecol Appl 20:1794-1800

Navarrete SA (1996) Variable predation: effects of whelks on a mid-intertidal successional community. Ecol

Editorial responsibility: Matthias Seaman,

Oldendorf/Luhe, Germany
Monogr 66:301-321

Olsen JL, Zechman FW, Hoarau G, Coyer JA, Stam WT, Valero M, Åberg P (2010) The phylogeographic architecture of the fucoid seaweed Ascophyllum nodosum: an intertidal 'marine tree' and survivor of more than one glacial-interglacial cycle. J Biogeogr 37:842-856

> Parmesan C, Gaines S, Gonzalez L, Kaufman DM, Kingsolver J, Peterson AT, Sagarin R (2005) Empirical perspectives on species' borders: from traditional biogeography to global change. Oikos 108:58-75

Pavia H, Toth GB (2000) Inducible chemical resistance to herbivory in the brown seaweed Ascophyllum nodosum. Ecology 81:3212-3225

Pavia H, Carr H, Åberg P (1999) Feeding preferences and habitat selection of three crustacean mesoherbivores inhabiting the brown seaweed Ascophyllum nodosum and its epiphytes. J Exp Mar Biol Ecol 236:15-32

Petraitis PS, Dudgeon SR (1999) Experimental evidence for the origin of alternate stable communities on rocky intertidal shores. Oikos 84:239-245

Petraitis PS, Latham RE, Niesenbaum RA (1989) The maintenance of species diversity by disturbance. Q Rev Biol 64: 393-418

Reed DC, Foster MS (1984) The effects of canopy shading on algal recruitment and growth in a giant kelp forest. Ecology 65:937-948

Schiel DR, Foster MS (2006) The population biology of large brown seaweeds: ecological consequences of multiphase life histories in dynamic coastal environments. Annu Rev Ecol Evol Syst 37:343-372

Schiel DR, Taylor DI (1999) Effects of trampling on a rocky intertidal algal assemblage in southern New Zealand. J Exp Mar Biol Ecol 235:213-235

Searle SR, Casella G, McCulloch CE (1992) Variance components. John Wiley \& Sons, New York, NY

Sousa WP (1984) The role of disturbance in natural communities. Annu Rev Ecol Syst 15:353-391

Svensson CJ, Pavia H, Åberg P (2009) Robustness in life history of the brown seaweed Ascophyllum nodosum (Fucales, Phaeophyceae) across large scales: effects of spatially and temporally induced variability on population growth. Mar Biol 156:1139-1148

> Taylor LR (1961) Aggregation, variance and the mean. Nature 189:732-735

> Underwood AJ, Jernakoff P (1981) Effects of interactions between algae and grazing gastropods on the structure of a low shore intertidal algal community. Oecologia 48: 221-223

Vadas RL, Wright WA, Millar SL (1990) Recruitment of Ascophyllum nodosum: wave action as a source of mortality. Mar Ecol Prog Ser 61:263-272

- Viejo RM, Åberg P, Cervin G, Lindegarth M (1999) The interactive effects of adult canopy, germling density and grazing on germling survival of the rockweed Ascophyllum nodosum. Mar Ecol Prog Ser 187:113-120

> Vucetich J, Waite T, Qvarnemark L, Ibargüen S (2000) Population variability and extinction risk. Conserv Biol 14: 1704-1714

Submitted: March 14, 2011; Accepted: July 28, 2011

Proofs received from author(s): October 6, 2011 